\title{
A review of current research on genetic factors associated with the functioning of the perceptual and emotional systems of the brain
}

\author{
Elena Vorobyeva $^{1}$, Fatima Hakunova $^{2}$, Irina Skirtach ${ }^{1,3}$, and Ekaterina Kovsh ${ }^{1,3 *}$ \\ ${ }^{1}$ Don state technical university, 344000, Rostov-on-Don, Russia \\ ${ }^{2}$ Adyghe State University, 352630, Maykop, Republic of Adygea, Russia \\ ${ }^{3}$ Southern federal university, 344006, Rostov-on-Don, Russia
}

\begin{abstract}
This article is a review of modern research on the relationship of genes of neurotransmitter, hormonal and neurotrophic systems of the brain with the work of perceptual and emotional systems of a person. The role of the BDNF, COMT, DRD2, HTR2A and other genes in the processes of visual recognition of words, virtual navigation, social perception, as well as in the implementation of other cognitive, emotional processes and executive functions, is discussed. The prospects of using the accumulated scientific data to study the characteristics of the visual perception of emotionally charged Internet-content with various informational orientations are discussed.
\end{abstract}

\section{Introduction}

The modern world is the world of information, and a person's success largely depends on how efficiently he selects relevant information from the irrelevant stream. The era of clip thinking poses new challenges for researchers. Due to the fact that for most modern people the visual modality is leading, understanding the mechanisms underlying the visual perception of information is an important task on the way to understanding the patterns of the functioning of the psyche as a whole, including understanding the contribution of biological factors. The emotional system of the brain performs a number of important functions (regulatory, protective, semantic, stimulating, etc.), being involved in various tasks, which indicates its high significance for humans. At the same time, the achievements of science make it possible to study the mechanisms of perception and emotional response in laboratory conditions, which are significantly different from natural ones, which imposes a significant limitation on the possibility of applying the acquired knowledge to a deep understanding of these processes. From the foregoing it follows that a significant layer of information remains unexplored. In order to get closer to understanding the mechanisms underlying the individual characteristics of mental processes, it is also necessary to take into account their genetic basis.

\footnotetext{
${ }^{*}$ Corresponding author: emkovsh@sfedu.ru
} 


\section{Genetic factors associated with visual recognition of words}

Visual recognition of words is one of the central topics in cognitive psychology and cognitive neurobiology. It is known that genetic factors affect the parameters of visual recognition of words, while the genes associated with this process are still not identified. Dopamine DRD2 receptor gene C957T polymorphism is associated with the availability and binding potential of the D2 receptor. Earlier studies have demonstrated the effect of this polymorphism on the perception and processing of verbal stimuli. The C957T polymorphism of the DRD2 gene is also associated with schizophrenia, with the C allele being a risk allele.

The results of a study of the influence of the DRD2 gene C957T polymorphism on the effectiveness of visual recognition of words by measuring its neuronal correlates and behavioral parameters are described in the article of Golimbet, V.E. with co-authors. Early (approximately $200 \mathrm{~ms}$ ) components of event-related potentials were recorded during the lexical solution of the task. 96 healthy subjects took part in the experiment, 53.1\% of which were men. Event-related brain potentials were recorded for words and pseudowords in implicit and explicit tasks. For analysis, two areas were selected in the left ventral temporal cortex, whose role in early visual processing of the text is well known. The results showed the main effect of the C957T polymorphism of the DRD2 gene on the amplitude of P200. Carriers of T/T genotype had higher amplitudes of $\mathrm{P} 200$ compared with carriers of the risk allele of schizophrenia C. Intragroup comparisons showed better ability to regulate attention to orthographic stimuli depending on job requirements and lexicality in the T/T group. The results of the study show that the C957T polymorphism of the DRD2 gene modulates the early stages of visual word recognition [1].

\section{Genetic factors associated with virtual navigation}

Mechanisms of visual perception and memory play a special role in the process of virtual navigation, providing parallel processing of spatial information. In a series of studies, a distinction was made between "spatial" navigation based on the hippocampus, which is based on knowledge of the relationship between landmarks in the environment for constructing a cognitive map and learning based on the habit of "response", which requires memorizing a number of action and is mediated by the caudate nucleus. Studies have shown that people spontaneously use one of these two alternative navigation strategies with almost equal frequency to solve this navigation problem, and this strategy correlates with the density of gray matter and brain activity recorded with using functional magnetic resonance imaging (fMRI). Scientists consider the Val66Met polymorphism of the neurotrophic brain factor BDNF gene as a possible inhibitor of hippocampal function.

Neurotrophic factor of the brain BDNF - a human protein encoded by the BDNF gene, refers to neurotrophins, substances that stimulate and support the development of neurons. BDNF acts on certain neurons of the central and peripheral nervous systems, helping to survive emerging neurons, increases the number and differentiation of new neurons and synapses. In the brain, it is active in the hippocampus, cortex, and forebrain, the areas responsible for learning and memory.

The results of a study of the Val66Met polymorphism of the BDNF gene' role in the implementation of virtual navigation and brain activation during the navigation task, using fMRI, are described in the article of Banner, H. et al. As a result, it was found that there is a genetic contribution to spontaneous strategies when carriers of the Met allele use the response strategy more often than individuals, homozygous for the Val allele. In addition, an increase in hippocampal activation was found in the group of carriers of the Val allele, 
compared with the group of carriers of the Met allele, during the execution of the virtual navigation task [2].

To date, a lot of data has been accumulated on the effect of the BDNF gene on the human emotional sphere $[3,4]$.

Jabbi M. et al. studied neurogenic mechanisms providing affective human education. In a high-order contextual study research, participants (33 Caucasian women of European descent at the age of $34.7 \pm 6.7$ years) genotyped by the Val66Met polymorphism of the neurotrophic factor BDNF gene should choose from the pair of images the one that most matches the emotion that the person expressed earlier the watched video, for the right choice, the participants were encouraged with a monetary reward. The fMRI method and genetic research were used. The fMRI revealed a blood-oxygen-level-dependent reactivity (BOLD) in the fronto-limbic region, which was associated with the Val66Met polymorphism of the BDNF gene at all three stages of the training task: aversive and predictable training, context-based decision making and choices related to monetary rewards and receipt result. Regarding the Val homozygous genotype, Met carriers showed a weakened prefrontal response of the ventromedial system to predictive affective signals, dorsolateral prefrontal signaling, which depended on the difficulty of decision-making, and increased prefrontal reactivity of the ventromedial system, which is associated with modulating the decision to avoid failure to complete the task and associated with this loss of money. The data obtained indicate that the Val66Met gene BDNF polymorphism is associated with the functional adjustment of behaviorally significant schemes of the frontolimbic region, especially with the participation of the ventromedial prefrontal cortex, during affective learning of a higher order [5].

The hereditary component of time perception mechanisms was studied by Bartholomew, A. J. et al. on a sample of 647 participants who were asked to recognize time periods in the range up to a second, and count time periods in the range above a second. A high correlation was shown between performance on a standardized cognitive battery and performance in time recognition tasks. The authors found the influence of ethnicity on the sense of time: African Americans and, possibly, Latin Americans differ in accuracy from other ethnic groups [6].

\section{Genetic factors associated with social perception}

Social perception is a special form of perception and an important prerequisite for successful social interaction. In the process of social perception, information is obtained about the behavior, thoughts and feelings of interaction partners. The complexity of this process requires monitoring by a variety of brain and genetic factors.

It's known that oxytocin and its receptor (OXTR) playing an important role in the processes of social perception and affiliation. Methylation (the epigenetic mechanism by which cells control transcription by modifying the chromatin structure) oxytocin DNA reduces OXTR gene expression, with high levels of methylation associated with autism spectrum disorders. According to the results of a study by Jack, A. et al., the level of methylation of oxytocin DNA can be used to predict individual variability in social perception [7].

Melchers, M. et al. conducted a study of the relationship between social perception abilities, measured by solving the interpersonal perception task, and polymorphism rs2268498 of the oxytocin receptor gene (OXTR; oxytocin is the peptide hormone of the paraventricular hypothalamic nucleus, which is transported to the posterior lobe of the pituitary gland, where deposited and secreted into the blood). In a study carried out with the involvement of 105 healthy participants, it was found that $\mathrm{T}$-allele carriers $(\mathrm{T} / \mathrm{T}$ and $\mathrm{T} / \mathrm{C}$ 
genotypes) demonstrate significantly higher efficiency in solving interpersonal perception tasks than carriers of the $\mathrm{C} / \mathrm{C}$ genotype [8].

Haram, M. et al. analyzed associations between the four oxytocin pathway genes (OXT, OXTR, AVP, and CD38) and the four areas of psychopathology associated with social behavior on a scale of positive and negative symptoms. For this, the polygenic risk score (PGRS) and single nucleotide polymorphisms of the OXTR gene (rs53576, rs237902 and rs2254298) were analyzed. The study included 734 patients with psychotic spectrum disorders according to DSM-IV and 420 healthy people. The presence of a reliable relationship between the symptom of emotional flattening and the A allele of polymorphism rs53576 of the OXTR gene was revealed [9].

\section{Genetic factors associated with other cognitive, emotional processes and executive functions}

The catechol-o-methyltransferase gene COMT is associated with the activity of the striopallid system and prefrontal areas [10] at the functional level, with aggressiveness [11], motivation [12], the success of the recognition of negative emotions [13] - on the psychological level.

Carriers of the Met allele of Val158Met polymorphism have a longer dopamine staying time in the prefrontal cortex, they also have a lower threshold of pain sensitivity, increased vulnerability to stress factors, and information processing are more efficient. In carriers of the Val allele, on the contrary, the duration of dopamine in the prefrontal cortex is lower due to the high activity of the enzyme catechol-O-methyltransferase; increased threshold of pain sensitivity, higher stress resistance [14].

An analysis of modern research on the topic showed the association of the Met allele of the BDNF and COMT genes with various adverse psychological characteristics: with a negative emotional background [15-17], with a high level of anticipatory anxiety [18]; and also with unfavorable features of the cognitive system: a decrease in the volume of shortterm and declarative memory [19], with a less successfully functioning error correction system during the search for solutions [20]. In the article of Altshuler, D. B. et al. it has been shown that carriers of highly active alleles of the BDNF, COMT, DRD2 genes suffering from oncological diseases of the nervous system have higher neuroplasticity, which is reflected in their faster return to work after surgery [21].

At the same time, some studies emphasize the relationship of the Met allele of the BDNF and COMT genes with a high level of attention switching during problem solving, multitasking [22], and higher executive functions [23-25].

The relationship of the COMT gene with the process of perception of information can be explained through a description of its influence on executive functions.

According to a study by Miranda, G. G. et al., conducted on a sample of 176 Americans aged 20-94 years, the COMT gene is associated with the thickness of the cerebral cortex in associative areas (prefrontal, parietal, cingular), in which the level of dopamine is high. In this regard, carriers of the Met allele have a thicker layer of the cortex in these areas, and, as a result, more efficient executive functions at different stages of ontogenesis (including in old age) [24]. Given the fact that the perception process includes both perceptual (detection, discrimination) and identification operations, we can assume that the COMT gene is associated with them due to its proven effect on executive functions.

A study of the neurobiological substrate underlying individual differences and personality traits, their significance for the emotional sphere, combined with the identification of the role of genes in the peripheral and central response to environmental influences, can give an idea of the physiological mechanisms involved in the regulation of homeostasis and the maintenance of physical and mental health. 
Genes play an important role in behavioral adaptation to environmental influences. More evidence is emerging illustrating the role of genes of the monoaminergic system in regulating the basic biological mechanisms of stress and emotions.

According to modern concepts, the process of cognition through selective attention can affect emotions, changing neural activity in the orbitofrontal cortex, as well as in the anterior cingulate gyrus. Preferences in decision making related to emotions are determined by genetic characteristics [26].

In the experiment of Weiss, E.M. et al. the relationships between the Val158Met polymorphism of the COMT gene, the 5-HTTLPR transporter gene of serotonin, and selfassessment data for the recognition and regulation of emotions were studied. Emotional competency was measured in a large sample of 289 healthy women using the Self-Assessed Emotional Ability Scale (SEAS). The highest self-esteem values of the effectiveness of regulation of emotions were obtained in carriers of the Met allele of the COMT gene, the lowest values for this indicator were found in women homozygous for the Val allele. Emotional recognition self-efficacy was found to be related to the 5-HTTLPR gene. Carriers of homozygous genotypes $(\mathrm{S} / \mathrm{S}$ and $\mathrm{L} / \mathrm{L})$ rated their ability to recognize emotions as less effective than carriers of the heterozygous S/L genotype [27]. In several studies, evidence was obtained that the polymorphism of the 5-HTTLPR serotonin transporter gene modulates emotionality and the risk of affective disorders. The short (S) allele has less functional effectiveness than the long (L) allele and is associated with increased emotional reactivity. One of the possible factors contributing to the high emotionality of carriers of the $\mathrm{S}$ allele may be the ineffective use of cognitive strategies, such as reassessment, to regulate emotional reactions.

537 people (384 women, 153 men; average age $=23.3$ years) took part in a study conducted by Sindermann, C. et al. in cognitive-behavioral approach. According to the results, the Met/Met genotype of the Val158Met polymorphism of the COMT gene is associated with lower awareness and higher severity of everyday cognitive errors but is not associated with attention control [28].

In the article of Blum $\mathrm{K}$. et al. it has been shown that individuals with insufficient serotonergic and / or dopaminergic receptors and an increased rate of synaptic dopamine catabolism due to the high activity of the COMT gene are prone to self-treatment with any substances or behavior that activate the release of dopamine, including alcohol, opiates, psychostimulants, nicotine, glucose, gambling, sex and abuse of Internet games [29], which may be due to the artificial attempt of carriers of the Val allele to activate brain structures that provide perceptual, emotional, cognitive processes.

An interesting article by Nogueira, N. G. et al., devoted to the study of the relationship of motor asymmetry with the COMT gene genotypes in a sample of right-handed Brazilian boys ( 27 people) and girls (15 people), whose average age was 25.12 years. Scientists have shown that carriers of the Met/Met genotype exhibit more pronounced interhemispheric asymmetry, while carriers of the Val/Met genotype exhibit more pronounced interhemispheric communication, neuroplasticity, and cognitive flexibility [30]. As is known, the profile of lateral organization affects the specifics of perceptual processes, and therefore, based on the results of Nogueira, N. G. et al., information processing strategies for carriers of different COMT gene genotypes may differ due to the predominance of intraor interhemispheric connections.

Jiang, W. et al. conducted a study on a sample of 753 high school students (516 girls, 237 boys), whose average age was 16.54 years. In their work it was shown that the COMT gene is associated with insight problem solving in men. Carriers of the Met allele demonstrated a significantly higher level of insight problem solving [31].

At the same time, these data did not find confirmation in the work of X. Yang et al., who did not receive a statistically significant relationship between the insight method of 
solving problems, gender, and COMT gene genotypes. The study participants were 425 healthy Chinese students ( 326 girls, 99 boys, the average age of which was 18.9 years). The presence of conflicting data may indicate a complex relationship between the structure of the polymorphic loci of the COMT gene and insight problem solving [32].

As known, the degree of susceptibility to impact (suggestibility) plays a special role in the duration of perceptual processes. The accumulated knowledge about the activity of different parts of the cerebral cortex of carriers of different COMT gene genotypes, as well as an understanding of the characteristics of the brain of people with varying degrees of suggestibility / hypnability [33] suggest a relationship between the Met/Met genotype and the high level of functioning of the prefrontal parts of the cerebral cortex, more thorough filtering, more successful detection and differentiation of relevant and irrelevant stimuli.

\section{Summary}

An analysis of the results of modern studies of genetic factors associated with the functioning of the perceptual and emotional systems of the brain showed a connection between the genotypes of the neurotransmitter, hormonal and neurotrophic systems of the brain and various features of visual recognition of words, virtual navigation, social perception and other cognitive, emotional processes and executive functions.

Supported by RFBR, project 18-29-22004

\section{References}

1. V.E. Golimbet, Z.V. Garakh, Y. Zaytseva, M.V. Alfimova, T.V. Lezheiko, N.V. Kondratiev, A.B. Shmukler, I.Y. Gurovich, V.B. Strelets, Neuropsychobiol., 76, 3, 143-150 (2018). DOI: 10.1159/000489712

2. H. Banner, V. Bhat, N. Etchamendy, R. Joober, V.D. Bohbot, Eur. J. of Neur. 33, 5, 968-977 (2011). DOI: 10.1111/j.1460-9568.2010.07550

3. E. Castrén, T. Rantamäki, CNS drugs, 24, 1, 1-7 (2010) https://doi.org/10.2165/11530010-000000000-00000

4. T.B. González-Castro, S. Pool-García, C. A. Tovilla-Zárate, I. E. Juárez-Rojop, M. L. López-Narváez, A. Frésan, H. Nicolini, Med., 98, 11, e14838, (2019) DOI: 10,1097 / MD.0000000000014838

5. M. Jabbi, B. Cropp, T. Nash, P. Kohn, J. S. Kippenhan, J. C. Masdeu, K. F. Berman, NeuroIm., 162, 373-383 (2017) doi: 10.1016/j.neuroimage.2017.08.080

6. A. J. Bartholomew, W. H. Meck, E. T. Cirulli, PLoS One, 10, 12, e0143873 (2015). doi: 10.1371/journal.pone.0143873

7. A. Jack, J.J Connelly, J.P. Morris, Neurosc. 6, 280, (2012) DOI: 10.3389/fnhum.2012.00280

8. M. Melchers, C. Montag, A. Felten, M. Reuter, Soc. Neurosc. 10, 4, 345-353 (2015). DOI: $10.1080 / 17470919.2015 .1008646$

9. M. Haram, M. Tesli, F. Bettella, S. Djurovic, O.A. Andreassen, Front. in hum. neurosc., 9, (2015). DOI: 10.3389/fnhum.2015.00009

10. L. M. Williams, J. M. Gatt, S. M. Grieve, C. Dobson-Stone, R. H. Paul, E. Gordon, P. $\begin{array}{llllll}\text { R. Schofield, } & \text { Neuroim. } & \text { 53, } & 3, & 918-925 & \text { (2010) }\end{array}$ https://doi.org/10.1016/j.neuroimage.2010.01.084

11. J. D. M. van Dongen, R. H. N. van Schaik, M. van Fessem, H. J. C. van Marle., Psych. of Viol., 8, 2, 269-276 (2018). http://dx.doi.org/10.1037/vio0000119

12. E. Aberg, A. Fandiño-Losada, L. K.Sjöholm, Y. Forsell, C. Lavebratt, J. of Aff.

Disord. 129, 1-3, 158-166 (2011). 
13. B. Gohier, C. Senior, J. Radua, W. El-Hage, A. Reichenberg, P. Proitsi, S. A. Surguladze, Eur. Psych. 29, 4, 197-202 (2014). https://doi.org/10.1016/j.eurpsy.2013.03.003

14. E.V. Vorobyeva, Psychogenetics (Publ. h. of the SFU, Rostov-on-Don, 2014)

15. T. Binbay, U. Kirli, E. Misir, H. Elbi, B. Kayahan, H. Onay, Alptekin, Turk. J. Psych. 29, 4 (2018)

16. L. M. Williams, J. M. Gatt, S. M. Grieve, C. Dobson-Stone, R. H. Paul, E. Gordon, P. $\begin{array}{lllll}\text { R. Schofield, } & \text { Neuroim. } & \mathbf{5 3}, & 3, & 918-925\end{array}$ https://doi.org/10.1016/j.neuroimage.2010.01.084

17. D. Kimhy, J. Vakhrusheva, Experience sampling in the study of psychosis. Experience Sampling in Mental Health Research. (Routledge, London, 2019) DOI: 10.4324/9781315398341-4

18. Z. Zou, J. Qiu, Y. Huang, J. Wang, W. Min, B. Zhou, Psych., health \& med. 24, 4, 505-511 (2019) https://doi.org/10.1080/13548506.2018.1516890

19. M. J. Spriggs, C. S.Thompson, D. Moreau, N. A. McNair, C. C. Wu, Y. N. Lamb, J. P. Hamm, Front. in hum. neurosc. 13, 22 (2019) https://doi.org/10.3389/fnhum.2019.00022

20. C. Beste, R. Willemssen, C. Saft, M. Falkenstein, Neuropsych. 48, 2, 366-373 (2010) https://doi.org/10.1016/j.neuropsychologia.2009.09.023

21. D. B. Altshuler, L. Wang, L. Zhao, Z. Miklja, J. Linzey, A. Brezzell, N. Gabel. NeuroOncology Practice, 20, 20, 1-11 (2019) https://doi.org/10.1093/nop/npy059

22. P. D. Gajewski, J. G. Hengstler, K., Golka, M. Falkenstein, C. Beste, Neurob. of Ag. 32, 12, 2327. e7-2327. e19. (2011)

23. B. G. Kurowski, B. Backeljauw, H. Zang, N. Zhang, L. J. Martin, V. Pilipenko, K. Yeates, J. head trauma rehab. 31, 3, E1 (2016) doi: $10.1097 /$ HTR.0000000000000162

24. G. G. Miranda, K. M. Rodrigue, K. M. Kennedy, Neurob. of ag. 73, 104-114 (2019) http://dx.doi.org/10.1016/j.neurobiolaging.2018.08.027

25. R. Parasuraman, B. Kidwell, R. Olmstead, M. K. Lin, R. Jankord, P. Greenwood, 56, 4, 760-771 (2014). https://doi.org/10.1177/0018720813510736

26. E. T. Rolls, Front. in hum. neurosc. 7, 74 (2013) DOI: 10.3389/fnhum.2013.00074

27. E. M. Weiss, H. H. Freudenthaler, A. Fink, E. M. Reiser, H. Niederstatter, S. Nagl, W. Parson, I. Papousek, J. intern. Neuropsych. Soc. 20, 5, 516-524 (2014) DOI: 10.1017/S135561771400023X

28. C. Sindermann, S. Markett, S. Jung, C. Montag, Mindful, 9, 5, 1479-1485 (2018) https://doi.org/10.1007/s12671-018-0893-4

29. K. Blum, Y. Liu, R. Shriner, M. S. Gold, Curr. Pharm. Des. 17, 12, 1158-1167 (2011) DOI: $10.2174 / 138161211795656819$

30. N. G. Nogueira, L. A. Fernandes, B. P. Ferreira, M. T. Batista, K. C. Alves, J. O. Parma, Perc. and mot. sk. 126, 3, 349-365 (2019) https://doi.org/10.1177/0031512519834738

31. W. Jiang, S. Shang, Y. Su, Front. in psych., 6, 1569 (2015) https://doi.org/10.3389/fpsyg.2015.01569

32. X. Yang, J. Zhang, S. Zhang, PeerJ, 7, 7:e6755 (2019) https://doi.org/10.7717/peerj.6755

33. A. V. Kirenskaya, Z. I. Storozheva, S. V. Solntseva, V. Y. Novototsky-Vlasov, M. N. Gordeev, Int. J. Clin. Exp. Hypn. 67, 1, 81-103 (2019) http://dx.doi.org/10.1080/00207144.2019.1553764 